\title{
The Influence of Different Grafting Materials on Alveolar Ridge Preservation: a Systematic Review
}

\author{
Jad Majzoub ${ }^{1}$, Andrea Ravida ${ }^{1}$, Thomas Starch-Jensen ${ }^{2}$, Mustafa Tattan ${ }^{3}$, Fernando Suárez-López \\ del $\mathrm{Amo}^{4}$ \\ ${ }^{1}$ Department of Periodontics and Oral Medicine, University of Michigan School of Dentistry, Ann Arbor, Michigan, USA. \\ ${ }^{2}$ Department of Oral and Maxillofacial Surgery, Aalborg University Hospital, Aalborg, Denmark. \\ ${ }^{3}$ Department of Periodontics, University of Iowa College of Dentistry, Iowa City, Iowa, USA. \\ ${ }^{4}$ Department of Periodontics, University of Oklahoma Health Sciences Center - College of Dentistry, Oklahoma City, \\ Oklahoma, USA.
}

\author{
Corresponding Author: \\ Fernando Suárez López del Amo \\ Department of Periodontics \\ University of Oklahoma Health Sciences Center \\ 1201 N Stonewall Ave, Oklahoma City, Oklahoma 73117 \\ USA \\ Phone: (405) 271-8001 \\ Fax: (405) 271-3794 \\ E-mail: fsuarezla@gmail.com
}

\begin{abstract}
Objectives: The purpose of the present review was to evaluate the effect of different bone substitutes used for alveolar ridge preservation on the post extraction dimensional changes.

Material and Methods: An electronic literature search in MEDLINE (PubMed), EMBASE (OVID) and Cochrane (CENTRAL) were performed, in addition to a manual search through all periodontics and implantology-related journals, up to December 2018. Inverse variance weighted means were calculated for all the treatment arms of the included trials for the quantitative analysis.

Results: Forty randomized controlled trials were included in the quantitative analysis. Dimensional changes were obtained from clinical measurements and three-dimensional imaging. The average amount of horizontal ridge resorption was 1.52 (SD 1.29) $\mathrm{mm}$ (allograft), 1.47 (SD 0.92) mm (xenograft), 2.31 (SD 1.19) mm (alloplast) and 3.1 (SD 1.07) mm for unassisted healing. Similarly, for all the evaluated parameters, the spontaneous healing of the socket led to higher bone loss rate than the use of a bone grafting material.

Conclusions: The utilization of a bone grafting material for alveolar ridge preservation reduces the resorption process occurring after tooth extraction. However, minimal differences in resorption rate were observed between allogeneic, xenogeneic and alloplastic grafting materials.
\end{abstract}

Keywords: alveolar bone atrophy; alveolar bone grafting; alveolar process atrophy; bone remodeling; evidence-based dentistry.

Accepted for publication: 5 September 2019

To cite this article:

Majzoub J, Ravida A, Starch-Jensen T, Tattan M, Suárez-López del Amo F.

The Influence of Different Grafting Materials on Alveolar Ridge Preservation: a Systematic Review

J Oral Maxillofac Res 2019;10(3):e6

URL: http://www.ejomr.org/JOMR/archives/2019/3/e6/v10n3e6.pdf

doi: $10.5037 /$ jomr.2019.10306 


\section{INTRODUCTION}

Adequate height and width of the alveolar hard and soft tissues is paramount importance for the placement of dental implants in a functionally and aesthetically optimal position [1]. However, following tooth extraction, the alveolar ridge undergoes physiological remodelling that results in vertical and horizontal osseous reduction, a increase in soft tissue thickness, and a narrowed band of keratinized mucosa $[\underline{2}, \underline{3}]$. These dimensional changes occur predominantly in the horizontal plane and are more pronounced during the first 3 months, followed by gradual reduction thereafter [4]. Previously published systematic reviews have demonstrated that a substantial loss of alveolar ridge volume following tooth extraction may compromise a future implant-supported fixed dental prosthesis $[\underline{5}, \underline{6}]$. Therefore, maintaining the post extraction dimensions will minimize the necessity for alveolar ridge augmentation prior to implant placement.

Alveolar ridge preservation (ARP) is a surgical technique that aims to minimize the degree of post extraction dimensional changes [7]. Various biomaterials, biologic agents, and technical approaches have been proposed. However, contradictory results, regarding the technique and/ or material of choice, have been reported. While a recent investigation considered the combination of a xenogenic or allogenic bone substitutes and resorbable collagen sponge or membrane as the most beneficial protocol, other investigations failed to identify a distinctly superior bone substitute when volumetric changes were in question $[\underline{8}, 9]$. On the other hand, most of the evidence supports the beneficial effect of ARP versus tooth extraction alone [10], concurring with many other previously published systematic reviews and meta-analyses [ $[\underline{8}, 11-16]$.

A reduction in the soft tissues accompanied by a narrowed band of keratinized mucosa may interfere with future peri-implant diseases [17]. Post extraction keratinized tissue dimensions subsequent to varying ARP techniques and biomaterials do not differ significantly from their changes following spontaneous extraction socket healing [9]. However, a randomized controlled trial revealed better preservation of the facial keratinized tissue after ARP using combination of corticocancellous porcine bone with a collagen barrier membrane [18].

Consequently, the scientific literature remains inconclusive with regard to the ideal surgical technique and biomaterial necessary to minimize post extraction dimensional changes of the alveolar ridge. Therefore, the aim of the present systematic review was to investigate the impact of different bone substitutes used for alveolar ridge preservation on the post extraction dimensional changes.

\section{MATERIAL AND METHODS Protocol and registration}

The methods of the analysis and inclusion criteria were specified in advance and documented in a protocol. The review was registered in PROSPERO. The present systematic review was conducted according to the Preferred Reporting Items for Systematic Reviews and Meta-Analysis (PRISMA) guidelines [10] and the Cochrane Handbook. Additionally, the Assessment of Multiple Systematic Reviews (AMSTAR) checklist was referenced to achieve the predetermined standards of reporting set for conducting systematic reviews [20].

\section{Focus question}

The following focus question was developed according to the population, intervention, comparison, and outcome (PICOS) design (Table 1):

What is the effect of different bone substitutes in ARP procedures performed in adult human subjects, when compared to unassisted and spontaneous healing of an extraction socket alone, on the prevention of alveolar ridge resorption performed in adult human subjects and reported in randomized controlled trials (RCTs)?

\section{Information sources}

An initial electronic systematic search was performed, without any publication date, language or journal restrictions, in the following electronic databases: National Library of Medicine (MEDLINE [PubMed] and ClinicalTrials.gov), EMBASE (OVID) and the Cochrane Central Register of Controlled Trials (CENTRAL).

\section{Search}

The following search strategy was designed for the MEDLINE (PubMed) database and then modified accordingly for other database engines: (socket[All Fields] AND ("preservation, biological"[MeSH Terms] OR ("preservation"[All Fields] AND "biological"[All Fields]) OR "biological preservation"[All Fields] OR "preservation"[All Fields])) OR (ridge[All Fields] AND ("preservation, biological"[MeSH Terms] OR ("preservation"[All 
Table 1. The focus question development according to the PICOS study design

\begin{tabular}{c|l}
\hline \multicolumn{1}{c|}{ Component } & \multicolumn{1}{c}{ Description } \\
\hline Population (P) & Subjects undergoing a tooth extraction \\
\hline Intervention (I) & $\begin{array}{l}\text { ARP using bone substitutes identified in the studies (i.e. an osseous allograft, xenograft and/or alloplast) with or without } \\
\text { employing a barrier membrane. }\end{array}$ \\
\hline Comparison (C) & Different bone substitutes \\
\hline Outcome (O) & $\begin{array}{l}\text { Dimensional stability of the alveolar ridge based on: } \\
\text { Primary: horizontal (bucco-lingual) and vertical (apico-coronal at mid-buccal and mid-lingual) socket dimensions } \\
\text { immediately after tooth extraction (baseline) and } \geq 3 \text { months after (follow-up). } \\
\text { Secondary: vertical bone level changes (at mesial and distal of the socket) and horizontal bone level changes (at several } \\
\text { subcrestal reference points). }\end{array}$ \\
\hline Study design (S) & Randomized controlled trial \\
\hline Focus question & $\begin{array}{l}\text { What is the effect of different bone substitutes in ARP procedures performed in adult human subjects, when compared } \\
\text { to unassisted and spontaneous healing of an extraction socket alone, on the prevention of alveolar ridge resorption } \\
\text { performed in adult human subjects and reported in randomized controlled trials (RCTs)? }\end{array}$ \\
\hline
\end{tabular}

Fields] AND "biological"[All Fields]) OR "biological preservation"[All Fields] OR "preservation"[All Fields])) AND Clinical Trial[ptyp]. The last search was performed on October of 2018. Additionally, to complement the electronic search process, an additional manual search, through the following relevant journals from January 2000 to December 2018, was performed to ensure a thorough screening assessment: "Journal of Periodontology", "Journal of Clinical Periodontology", "Clinical Oral Implants Research", "Clinical Implant Dentistry and Related Research", "Journal of Dental Research", "International Journal of Oral and Maxillofacial Implants", "International Journal of Oral and Maxillofacial Surgery", and the "International Journal of Periodontics and Restorative Dentistry". The bibliographies of the retrieved studies and previous published reviews on the topic were also searched for potential articles.

\section{Selection of studies}

After the primary systematic search, all the titles and abstracts were scanned independently by two investigators (JM and AR), followed by the full-text assessment of the potentially eligible studies. In case of any doubt or disagreement between the two authors regarding study selection, a third investigator (FS) was contacted.

\section{Types of publications}

Only human randomized clinical trials have been included. Non-randomized clinical trial studies such as prospective controlled clinical studies, case series, case reports, and retrospective studies were excluded, furthermore, letters, editorials, $\mathrm{PhD}$ theses were not considered.

\section{Types of studies}

The included group must have involved utilization of a single bone graft material (no combination of different bone substitutes materials), or spontaneous healing sockets.

\section{Types of participants/population}

Subjects, in which changes in the outcome measures (alveolar ridge dimensions) were assessed either clinically or with the use of three-dimensional radiography with standardization.

\section{Inclusion and exclusion criteria}

A systematic literature search limited to RCTs, without any language restriction, was performed based on the following criteria: studies having recruited a minimum of 5 healthy adult individuals ( $\geq 18$ years old) per study arm who had undergone at least one tooth extraction, while allowing for at least 2 months of healing. The inclusion of a control group (spontaneous socket healing) was not considered necessary to be selected for inclusion. Hence, comparative studies may or may not have included a control group (unassisted socket healing). The approach for the intervention must have involved the utilization of a bone substitute (whether or not it was covered with a barrier membrane) without any additional therapy that may have interfered with the healing outcomes (e.g. growth factors, platelet-rich plasma, immediate implants etc.). The changes in the alveolar ridge dimensions must have been measured either clinically or with the use of three-dimensional radiography that is standardized between visits. Thus, studies that have not assessed clinical outcomes, or those that performed 
two-dimensional radiographic assessment of the ridge dimensions were excluded. All non-randomized studies (i.e. prospective controlled and non-controlled, case series, case reports and retrospective study designs) were also not included. The corresponding authors of potentially eligible studies were contacted, to clarify any uncertainties, ahead of making a final decision. In the absence of a response and/or if the data was insufficient, the study was excluded from the final review.

\section{Data extraction}

The data were separately extracted by two investigators (AR and $\mathrm{TM}$ ) according to the aforementioned criteria to confirm the suitability of each trial. In case of any discrepancies during the data extraction, a third investigator (FS) was referred to for resolution of the matter. The collected data consisted of the following:

- General study characteristics (date and country of publication, participants' characteristics, number of groups/interventions, and study setting).

- Clinical procedures (bone substitute, membrane type, type of surgical procedure and type of extraction (i.e. flapped versus flapless), and follow-up/healing time).

- Quantitative dimensional changes of the extraction socket.

- Source of funding (e.g. institutional, commercial, self-funded).

\section{Data items}

Data were collected and arranged from selected articles in the following fields:

- "Year"- describes the date of publication.

- "Study design " - indicates if the patients were divided in a parallel or split-mouth design.

- "Ridge preservation" - describes a procedure to reduce alveolar bone loss after tooth extraction.

- "Material used in alveolar ridge preservation" - indicates the type of bone graft substitutes (if present) used to restore the damaged extraction socket after tooth extraction.

- "Clinical and radiographic parameters" - revealed the changes in alveolar dimensions during the socket healing process.

\section{Risk of bias within studies}

For assessing the quality of the included trials, the same authors (JM and AR), individually examined and categorized the studies according to The Cochrane
Risk of Bias Tool for Randomized Controlled Trials [21]. The risk of bias was considered low if a study provided information on all the parameters. A study that had not provided information on even one of the parameters was considered as having a moderate risk of bias, and if a trial or article lacked information about 2 or more parameters, it was categorized as having a high risk of bias.

\section{Statistical analysis}

Inverse variance weighted means were calculated for all the treatment arms of the included trials for the quantitative analysis to display the amount (in $\mathrm{mm}$ ) of ridge resorption in all available and measured dimensions. Ridge resorption of the control groups (unassisted socket healing) was also calculated similarly. Data were expressed as means with standard deviations and all statistical analyses were performed using Rstudio for Macintosh (Rstudio Version 1.1.383, Rstudio, Inc., Massachusettes, USA) and the metafor package.

\section{RESULTS \\ Study selection}

The initial search yielded a total of 1246 studies, from which 549 were excluded subsequent to duplicate removal. Seventeen additional records were identified through direct hand-search of the references and journals. After screening 714 titles and abstracts, 85 studies remained for full-text examination. After thorough evaluation of the studies according to the eligibility criteria, 40 RCTs were included in the quantitative analysis [22-61]. The most frequent reasons for exclusion of the articles were due to:

- the use of biologics, growth factors or healing enhancers, volumetric analyses;

- a histological study design short of clinical data on ridge dimensions;

- immediate implant placement or alternative protocols not within the scope of this review.

Figure 1 displays the screening process leading to the selection of the included 40 trials and data S1 tabulates the causes for extraction of the articles.

\section{Study characteristics}

All articles selected for the quantitative analysis reported results of RCTs aimed at evaluating the effect of different bone substitutes on decreasing post extraction alveolar ridge atrophy. All studies except Azizi and Moghaddam [23], that is in Farsi, 
were published in the English language. Seven studies were performed in a split-mouth manner [36,52-57], while the rest employed a parallel arm design [2251, 58-61]. Four of the total studies included [ 61] more than 2 treatment arms, while the remaining trials consisted of one comparative treatment group [22-57]. The follow-up time of the included studies ranged from 3 to 8 months. Excluding two multi-center studies $[39,60]$, in Italy and Spain, all the trials were conducted at a single center. The year of publication ranged from 2003 to 2018 .

The selection of the 40 trials rendered the inclusion of 1178 subjects (age range from 18 to 81 years old) with a total of 1366 extraction sockets for analysis.
Thirty studies performed ARP exclusively on nonmolar extraction sockets $[22-24,26,27,29-31,33,35,37-$ $\underline{41}, \underline{44-57,61]}$, while the rest included molar sockets as well $[25, \underline{28}, \underline{32}, \underline{36}, \underline{42}, \underline{43}, \underline{58-60}]$. Information regarding the type of teeth was not available in one article [34]. Except for 11 studies which had utilized threedimensional radiography for measurement acquisition $[27,35-37,39-43,56,57]$, the outcome measures were taken clinically using a custom-made template for the all other studies [2-26, $\underline{28-34}, \underline{38}, \underline{44-55}, \underline{58-61]}$.

Two studies $[\underline{28}, \underline{39}]$ were performed at a private practice setting only, $2[\underline{23}, \underline{60}]$ were carried out at both institutional and private practice settings

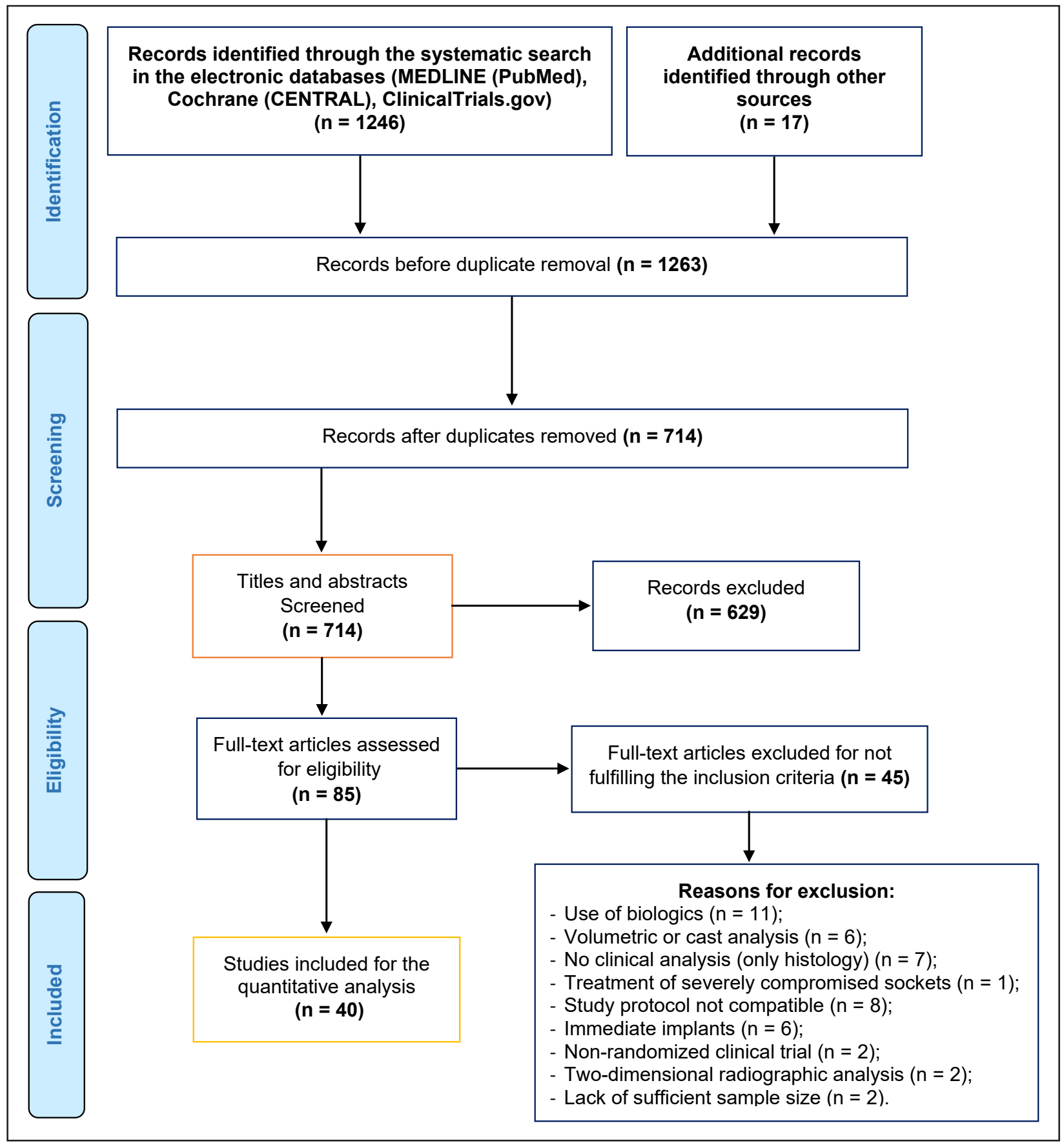

Figure 1. Flowchart of literature search and selection process. 
and the remainder were conducted at an institutional setting only [22,24-27,29-38,40-59,61]. Detailed characteristics of the included RCTs are presented in Table 2.

\section{Allograft}

Sixteen articles, consisting of a total of 394 treated extraction sockets $[\underline{26}, \underline{27}, \underline{30-33}, \underline{41}, \underline{44-46}, \underline{49-52}, \underline{56}, \underline{61}]$ had reported the use of an allogeneic bone substitute in their study for ARP. The average amount of horizontal ridge resorption was 1.52 (SD 1.29) $\mathrm{mm}$, whereas the loss of ridge height amounted to 0.68 (SD 0.66) and 0.65 (SD $1.29 \mathrm{~mm}$ at the mid buccal and mid lingual sites, respectively. In addition, based on two studies that evaluated the horizontal resorption at several reference points below the crest $[\underline{45}, \underline{56}]$, grafted sockets lost an average of 2.75 (SD 2.05), 1.93 (SD 1.62) and 0.75 (SD 0.79) $\mathrm{mm}$ at reference points 1, 3 and $5 \mathrm{~mm}$ below the crest. Finally, based on the 2 studies that evaluated the changes in ridge height adjacent to the extraction socket $[33,45]$, augmented sockets lost 0.3 (SD 0.55) and 0.45 (SD 0.5) $\mathrm{mm}$ at the mesial and distal aspects, respectively.

\section{Xenograft}

Twenty-two studies, consisting of 455 treated extraction sockets, utilized a xenogeneic bone substitute [23-25, 28, 29, $\underline{34-}$ $40,42,43,46,48,51,54,55,58-60]$. On average, the amount of reported resorption in the horizonal dimension was 1.47 (SD 0.92) $\mathrm{mm}$, whereas the loss of ridge height amounted to 0.68 (SD 1.04) and 0.47 (SD 0.97) $\mathrm{mm}$ at the mid buccal and mid lingual sites, respectively. Based on the studies that evaluated the horizontal ridge resorption below the crest $[34,36,37,39,40,43]$, grafted sockets lost 0.91 (SD 1.46), 0.66 (SD 0.72) and 0.41 (SD 0.58) mm at reference points 1,3 and $5 \mathrm{~mm}$ below the crest, respectively.

\section{Alloplast}

Seven studies, including 103 sockets reported the use of an osseous alloplast $[\underline{22}, \underline{35}, \underline{38}, \underline{47}, \underline{53}, \underline{55}, \underline{58}]$. The resorption in the horizontal dimension based on the aforementioned studies was 2.31 (SD 1.19) $\mathrm{mm}$. For the resorption in the vertical plane, only three articles $[35,38,53]$ reported this measurement and reporting a loss of $1.23(\mathrm{SD} 1.84) \mathrm{mm}$ at the mid buccal site and 1.07 (SD 0.91) $\mathrm{mm}$ at the mid lingual site. Only 1 of the 6 studies [35] assessed the changes in the horizontal dimension at reference points below the crest (3.1 [SD 1.6] and 5.7 [SD 3] $\mathrm{mm}$ at 3, and $6 \mathrm{~mm}$ below the ridge, respectively). The reported vertical bone resorption on the mesial and distal aspects were also measured by one of the studies

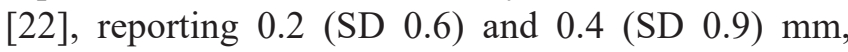
respectively.

\section{Unassisted socket healing}

Fifteen studies included a total of 161 post extraction sockets that were left to heal without any intervention or addition of a bone substitute $[\underline{22-24}, \underline{27}, \underline{28}, \underline{33-}$ $36,42,54,57-60]$. The subsequent resorption was 3.1 (SD 1.07) $\mathrm{mm}$ in the horizontal dimension, 1.79 (SD 0.98$) \mathrm{mm}$ in the mid buccal vertical dimension and 1.53 (SD 1.02) $\mathrm{mm}$ in the mid lingual vertical dimension. Based on studies that further evaluated other parameters of socket healing [ㄹ-36, 57], there was 2.98 (SD 2.01) $\mathrm{mm}$ of horizontal ridge resorption $1 \mathrm{~mm}$ below the crest, 1.59 (SD 1.23) $\mathrm{mm}$ at $3 \mathrm{~mm}$ below the crest and 0.96 (SD 0.69) $\mathrm{mm}$ at $5 \mathrm{~mm}$ below the crest. Regarding the ridge height on the mesial and distal areas, an average resorption of 0.52 (SD 0.85) and 0.57 (SD 0.93) $\mathrm{mm}$ was reported, respectively $[22, \underline{23}, \underline{25}, \underline{33}, \underline{34}, \underline{52}$ -54].

\section{Quality assessment}

The adopted risk of bias assessment for the included RCTs, for criteria and method of reporting, was according to the recommendations of The Cochrane Risk of Bias Tool for Randomized Controlled Trials [21] (Table 3). Accordingly, 4 articles were considered to be at a low risk of bias $[\underline{37-39}, \underline{41}], 16$ at a moderate risk of bias $[\underline{24-27}, \underline{\underline{4}-36}, \underline{40}, \underline{44}, \underline{49-52}, \underline{55}, \underline{56}, \underline{61}]$, and 20 at a high risk of bias $[22,23,28-36,40,44,49-$

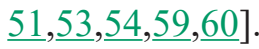

\section{DISCUSSION}

The clinical benefits of ARP have been extensively demonstrated and robustly evidenced [7]. To date, a plethora of scientific evidence, consisting of many clinical trials and meta-analyses, have repeatedly shown an attenuated magnitude of ridge resorption with ARP through a diverse set of protocols and techniques $[\underline{8}, 9,12,62]$. The results from this present analysis corroborate previous studies when demonstrating an average horizontal resorption rate of 3.4 (SD 1.07) $\mathrm{mm}$ for unassisted socket healing, compared to an average 1.43 (SD 0.89) $\mathrm{mm}, 1.52$ (SD 1.29) $\mathrm{mm}$ and 1.84 (SD 1.08) $\mathrm{mm}$ 


\begin{tabular}{|c|c|c|c|c|c|c|c|c|c|c|c|c|c|}
\hline Study & $\begin{array}{c}\text { Year of } \\
\text { publication }\end{array}$ & $\begin{array}{l}\text { Study } \\
\text { design }\end{array}$ & \begin{tabular}{|c|}
$\begin{array}{c}\text { Allowed } \\
\text { healing } \\
\text { time } \\
\text { (months) }\end{array}$ \\
\end{tabular} & $\begin{array}{c}\mathbf{N} \text { patients } \\
\text { (group 1/ } \\
\text { group 2) }\end{array}$ & $\begin{array}{l}\text { N sockets } \\
\text { (group 1/ } \\
\text { group 2) }\end{array}$ & $\begin{array}{c}\text { Inclusion } \\
\text { of molar } \\
\text { teeth? }\end{array}$ & $\begin{array}{c}\text { Bone substitute materials } \\
\text { used }\end{array}$ & $\begin{array}{c}\text { Type } \\
\text { of bone } \\
\text { substitutes }\end{array}$ & $\begin{array}{c}\text { Barrier } \\
\text { membrane } \\
\text { used }\end{array}$ & $\begin{array}{l}\text { Flap/primary } \\
\text { closure }\end{array}$ & Setting & Country & $\begin{array}{l}\text { Method of } \\
\text { measurement }\end{array}$ \\
\hline Aimetti et al. [22] & 2009 & Parallel & 3 & $22 / 18$ & $22 / 18$ & No & MGCSH/nothing & Alloplast & None & $\mathrm{No} / \mathrm{no}$ & University & Italy & Clinical \\
\hline $\begin{array}{l}\text { Azizi and } \\
\text { Moghaddam [23] }\end{array}$ & 2009 & Parallel & 6 & $15 / 15$ & $15 / 15$ & NR & DBBM/nothing & Xenograft & $\begin{array}{l}\text { Collagen } \\
\text { /none }\end{array}$ & Yes/yes & $\begin{array}{c}\text { University } \\
\text { and private } \\
\text { practice }\end{array}$ & Iran & Clinical \\
\hline Barone et al. [24] & 2008 & Parallel & 7 & $20 / 20$ & $20 / 20$ & No & CCPB/nothing & Xenograft & $\begin{array}{l}\begin{array}{c}\text { Collagen } \\
\text { /none }\end{array} \\
\end{array}$ & Yes/yes & University & Italy & Clinical \\
\hline Barone et al. [25] & 2014 & Parallel & 3 & $30 / 29$ & $32 / 32$ & Yes & ССРВ & Xenograft & Collagen & $\mathrm{No} /$ no & $\begin{array}{c}\text { University } \\
\text { and private } \\
\text { practice }\end{array}$ & Italy & Clinical \\
\hline Borg et al. [26] & 2015 & Parallel & 5 & $20 / 20$ & $20 / 20$ & No & \begin{tabular}{|c|}
$100 \%$ FDBA \\
$/ 70 \%$ cortical mineralized and \\
$30 \%$ cortical
\end{tabular} & Allograft & d-PTFE & Yes/yes & University & USA & Clinical \\
\hline $\begin{array}{l}\text { Brownfield and } \\
\text { Weltman [27] } \\
\end{array}$ & 2012 & Parallel & 3 & 17 (total) & $10 / 10$ & No & \begin{tabular}{|c|}
$\begin{array}{c}\text { DBM with cancellous bone } \\
\text { chips/nothing }\end{array}$ \\
\end{tabular} & $\begin{array}{c}\begin{array}{c}\text { Allograft/ } \\
\text { nothing }\end{array} \\
\end{array}$ & None & $\mathrm{No} /$ no & University & USA & $\mathrm{CBCT}$ \\
\hline $\begin{array}{l}\text { Cardaropoli et al. } \\
{[28]}\end{array}$ & 2014 & Parallel & 4 & 41 (total) & $24 / 24$ & Yes & $\begin{array}{l}\text { DBBM blended with } \\
\text { collagen/nothing }\end{array}$ & $\begin{array}{c}\text { Xenograft/ } \\
\text { nothing }\end{array}$ & Collagen/none & No/no & $\begin{array}{l}\text { Private } \\
\text { practice }\end{array}$ & Italy & Clinical \\
\hline $\begin{array}{l}\text { Cook and Mealey } \\
\text { [29] }\end{array}$ & 2013 & Parallel & 5 & 22 & 23 & No & $\begin{array}{c}90 \% \text { inorganic bovine }+10 \% \\
\text { porcine collagen fibers }\end{array}$ & Xenograft & Collagen & Yes/yes & University & USA & Clinical \\
\hline $\begin{array}{l}\text { Eskow and Mealey } \\
{[30]}\end{array}$ & 2014 & Parallel & 5 & 32 (total) & $15 / 17$ & No & FDBA CO/FDBA CA & \begin{tabular}{|c|} 
Allograft/ \\
allograft
\end{tabular} & \begin{tabular}{|c|}
$\begin{array}{c}\text { Collagen } \\
\text { (if dehiscence) }\end{array}$ \\
\end{tabular} & No & University & USA & Clinical \\
\hline Fotek et al. [31] & 2009 & Parallel & 4 & $8 / 10$ & $8 / 10$ & No & $\begin{array}{c}\text { Solvent-preserved mineralized } \\
\text { cancellous allograft }\end{array}$ & Allograft & ADM/d-PTFE & No & University & USA & Clinical \\
\hline Hoang et al. [32] & 2012 & Parallel & 4 and 5 & $16 / 14$ & $16 / 14$ & Yes & $\begin{array}{c}\text { DBM putty with one size of } \\
\text { bone particles/DBM putty } \\
\text { with two different sizes of } \\
\text { bone particles }\end{array}$ & $\begin{array}{c}\text { Allograft } \\
\text { allograft }\end{array}$ & $\begin{array}{c}\text { Collagen } \\
\text { membrane } \\
\text { (if dehiscence) }\end{array}$ & \begin{tabular}{|c|}
$\begin{array}{c}\text { Flap was reflected } \\
\text { only if a significant } \\
\text { bony dehiscence } \\
\text { was detected }\end{array}$ \\
\end{tabular} & University & USA & Clinical \\
\hline Iasella et al. [33] & 2003 & Parallel & 4 or 6 & $12 / 12$ & $12 / 12$ & No & $\begin{array}{c}\text { Tetracycline hydrated FDBA } \\
\text { /nothing }\end{array}$ & $\begin{array}{c}\text { Allograft/ } \\
\text { nothing }\end{array}$ & Collagen/none & Yes/yes & University & USA & Clinical \\
\hline $\begin{array}{l}\text { Iorio-Siciliano et } \\
\text { al. [34] }\end{array}$ & 2017 & Parallel & 6 & $10 / 10$ & $10 / 10$ & $\mathrm{~N} / \mathrm{R}$ & $\begin{array}{l}\text { Bovine-derived xenograft } \\
\text { collagen/nothing }\end{array}$ & $\begin{array}{c}\text { Xenograft } / \\
\text { nothing }\end{array}$ & Collagen/none & Yes/yes & University & Italy & Clinical \\
\hline Jung et al. [35] & 2013 & Parallel & 6 & $10 / 10 / 10 / 10$ & $10 / 10 / 10 / 10$ & No & $\begin{array}{c}\text { B-TCP/DBBM-C/DBBM-C/ } \\
\text { nothing }\end{array}$ & $\begin{array}{c}\text { Alloplast/ } \\
\text { xenograft/ } \\
\text { xenograft/ } \\
\text { nothing }\end{array}$ & $\begin{array}{c}\text { None/collagen } / \\
\text { none/none }\end{array}$ & No & University & Switzerland & $\mathrm{CBCT}$ \\
\hline Jung et al. [36] & 2018 & \begin{tabular}{|c|} 
Split- \\
mouth
\end{tabular} & 6 & 18 & $18 / 18$ & Yes & DBBM-C/nothing & $\begin{array}{c}\text { Xenograft/ } \\
\text { nothing }\end{array}$ & Collagen/none & No & University & China & $\mathrm{CBCT}$ \\
\hline Lim et al. [37] & 2017 & Parallel & 4 & 26 & 26 & No & Collagenated bovine bone & Xenograft & Collagen & Yes & University & Korea & CBCT \\
\hline Mardas et al. [38] & 2010 & Parallel & 8 & $13 / 14$ & $13 / 14$ & No & $\mathrm{DBBM} /$ bone ceramic & $\begin{array}{c}\text { Xenograft } / \\
\text { alloplast }\end{array}$ & Collagen & Yes/yes & University & England & Clinical \\
\hline Meloni et al. [39] & 2015 & Parallel & 5 & $15 / 15$ & $15 / 15$ & No & DBBM & Xenograft & None & No & $\begin{array}{l}\text { Private } \\
\text { practice }\end{array}$ & Italy, Spain & CBCT \\
\hline Nart et al. [40] & 2017 & Parallel & 5 & 21 (total) & $11 / 11$ & No & DBBM/DBBM-C & $\begin{array}{c}\text { Xenograft/ } \\
\text { xenograft }\end{array}$ & Collagen & No & University & Spain & $\mathrm{CBCT}$ \\
\hline Natto et al. [41] & 2017 & Parallel & 4 & $14 / 14$ & $14 / 14$ & No & $\begin{array}{c}\text { FDBA and collagen matrix } \\
\text { seal/FDBA and collagen } \\
\text { sponge }\end{array}$ & $\begin{array}{c}\text { Allograft } \\
\text { allograft }\end{array}$ & None & No & University & USA & CBCT \\
\hline Pang et al. [42] & 2014 & Parallel & 6 & $15 / 15$ & $15 / 15$ & Yes & DBBM/nothing & $\begin{array}{c}\text { Xenograft } \\
\text { /nothing }\end{array}$ & Collagen/none & Yes/NR & University & China & $\mathrm{CBCT}$ \\
\hline Park et al. [43] & 2016 & Parallel & 4 & 14 & 14 & Yes & $\begin{array}{l}\text { Demineralized bovine bone } \\
\text { matrix mixed with } 10 \% \\
\text { collagen }\end{array}$ & Xenograft & Collagen & No & University & Korea & CBCT \\
\hline Parashis et al. [44] & 2016 & Parallel & 4 & 23 & 23 & No & FDBA & Allograft & Collagen & No & University & USA & Clinical \\
\hline Poulias et al. [45] & 2013 & Parallel & 4 & 12 & 12 & No & \begin{tabular}{|l|} 
Mineralized, CA, particulate \\
\end{tabular} & Allograft & Polylactide & Yes/yes & University & USA & Clinical \\
\hline Sadeghi et al. [46] & 2016 & Parallel & $4-6$ & $10 / 10$ & $10 / 10$ & No & DFDBA/DBBM & $\begin{array}{l}\text { Allograft/ } \\
\text { xenograft } \\
\end{array}$ & Collagen & Yes & University & Iran & Clinical \\
\hline Toloue et al. [47] & 2012 & Parallel & 3 & 12 & 13 & No & Calcium sulfate & Alloplast & None & No & University & USA & Clinical \\
\hline Vance et al. [48] & 2004 & Parallel & 4 & 12 & 12 & No & DBBM & Xenograft & Collagen & Yes & University & USA & Clinical \\
\hline Whetman et al. [49] & 2016 & Parallel & $\left|\begin{array}{c}2-2.5 \\
\text { or } 4.5-5\end{array}\right|$ & $22 / 19$ & $22 / 19$ & No & DFDBA & Allograft & \begin{tabular}{|c|} 
Collagen \\
(if dehiscence)
\end{tabular} & Yes & University & USA & Clinical \\
\hline $\begin{array}{l}\text { Wood and Mealey } \\
\text { [50] }\end{array}$ & 2012 & Parallel & 5 & $16 / 16$ & $16 / 16$ & No & DFDBA/FDBA & \begin{tabular}{|c|} 
Allograft/ \\
allograft
\end{tabular} & Collagen & No & University & USA & Clinical \\
\hline $\begin{array}{l}\text { Serrano Mendez } \\
\text { [51] }\end{array}$ & 2017 & Parallel & 6 & $10 / 10$ & $10 / 10$ & No & DFDBA/DBBM & $\begin{array}{c}\text { Allograft/ } \\
\text { xenograft }\end{array}$ & Collagen & Yes/yes & University & Columbia & Clinical \\
\hline Fernandes et al. [52] & 2016 & \begin{tabular}{|c|}
$\begin{array}{c}\text { Split- } \\
\text { mouth }\end{array}$ \\
\end{tabular} & $6-8$ & 16 & 16 & No & Mineralized bone graft & Allograft & $\mathrm{ADM}$ & No & University & Brazil & NR \\
\hline Fernandes et al. [53] & 2011 & $\begin{array}{l}\text { Split- } \\
\text { mouth }\end{array}$ & 6 & 18 & 18 & No & $\begin{array}{c}\text { Anorganic bone matrix with } \\
\text { synthetic cell-binding peptide } \\
\text { P-15 }\end{array}$ & Alloplast & $\mathrm{ADM}$ & No & University & Brazil & Clinical \\
\hline Festa et al. [54] & 2013 & $\begin{array}{c}\text { Split- } \\
\text { mouth }\end{array}$ & 6 & $15 / 15$ & $15 / 15$ & No & CCPB/nothing & $\begin{array}{c}\text { Xenograft } / \\
\text { nothing }\end{array}$ & $\begin{array}{c}\text { Soft cortical } \\
\text { membrane/ } \\
\text { none }\end{array}$ & Yes/yes & University & Italy & Clinical \\
\hline Gholami et al. [55] & 2012 & \begin{tabular}{|c|} 
split- \\
mouth
\end{tabular} & $\begin{array}{c}6.9(\mathrm{SD} \\
0.8)\end{array}$ & 12 & $14 / 14$ & No & $\begin{array}{c}\text { DBBM/nanocrystalline } \\
\text { hydroxyapatite }\end{array}$ & $\begin{array}{c}\text { Xenograft/ } \\
\text { alloplast }\end{array}$ & Collagen & Yes/yes & University & Iran & Clinical \\
\hline Hassan et al. [56] & 2017 & $\begin{array}{l}\text { Split- } \\
\text { mouth }\end{array}$ & 3 & 9 & $11 / 11$ & No & $\begin{array}{c}\text { Demineralized freeze-dried } \\
\text { bone/mineralized freeze-dried } \\
\text { bone }\end{array}$ & $\begin{array}{c}\text { Allograft/ } \\
\text { allograft }\end{array}$ & $\begin{array}{l}\text { Amnion- } \\
\text { chorion/d- } \\
\text { PTFE } \\
\end{array}$ & $\mathrm{No} /$ no & University & USA & $\begin{array}{l}\text { Clinical and } \\
\text { CBCT }\end{array}$ \\
\hline $\begin{array}{l}\text { Temmerman et al. } \\
\text { [57] }\end{array}$ & 2016 & \begin{tabular}{|r|} 
Split- \\
mouth
\end{tabular} & 3 & 22 & 22 & No & Nothing & Nothing & None & No & University & Belgium & $\mathrm{CBCT}$ \\
\hline Kotsakis et al. [58] & 2014 & Parallel & 5 & $10 / 8 / 6$ & $12 / 12 / 6$ & Yes & $\begin{array}{c}\text { Calcium phosphosilicate putty } \\
\text { alloplast/bovine bone mineral/ } \\
\text { nothing }\end{array}$ & $\begin{array}{c}\text { Alloplast/ } \\
\text { xenograft/ } \\
\text { nothing }\end{array}$ & None & No & University & USA & Clinical \\
\hline Guarnieri et al. [59] & 2017 & Parallel & 4 & $8 / 9$ & $8 / 9$ & Yes & Porcine-derived bone/nothing & $\begin{array}{c}\text { Xenograft/ } \\
\text { nothing }\end{array}$ & Collagen/none & $\mathrm{No} /$ no & University & Italy & Clinical \\
\hline Barone et al. [60] & 2017 & Parallel & 3 & $30 / 30 / 30$ & $30 / 30 / 30$ & Yes & $\begin{array}{c}\text { Collagenated CCPB/cortical } \\
\text { porcine bone/nothing }\end{array}$ & $\begin{array}{c}\text { Xenograft/ } \\
\text { xenograft/ } \\
\text { nothing }\end{array}$ & $\begin{array}{c}\text { Collagen/ } \\
\text { collagen/none }\end{array}$ & No/no/no & University & Italy, Spain & Clinical \\
\hline Demetter et al. [61] & 2017 & Parallel & 5 & 58 (total) & $19 / 19 / 20$ & No & $\begin{array}{c}100 \% \text { cortical FDBA } / 100 \% \\
\begin{array}{c}\text { CA/FDBA } 50-50 \% \text { cortico- } \\
\text { cancellous FDBA }\end{array}\end{array}$ & \begin{tabular}{|c|} 
Allograft/ \\
allograft/ \\
allograft \\
\end{tabular} & d-PTFE & No & University & USA & Clinical \\
\hline
\end{tabular}

$\mathrm{N}=$ number, $\mathrm{d}-\mathrm{PTFE}=$ dense polytetrafluoroethylene $\mathrm{MGCSH}=$ medical-grade calcium sulfate hemihydrate DBM $=$ demineralized bone matrix DBBM $=$ deproteinized bovine bone mineral; $\mathrm{DBBM}-\mathrm{C}=$ deproteinized bovine bone mineral with $10 \%$ collagen; $\mathrm{CCPB}=$ cortico-cancellous porcine bone; FDBA = freeze-dried bone allograft; $\mathrm{NR}=$ not reported; $\mathrm{CBCT}=$ cone-beam computed tomography. 
Table 3. Risk of bias assessment for the included randomized controlled trials (according The Cochrane Risk of Bias Tool for Randomized Controlled Trials) [21]

\begin{tabular}{|c|c|c|c|c|c|c|c|c|}
\hline Study & $\begin{array}{c}\text { Random } \\
\text { sequence } \\
\text { generation }\end{array}$ & $\begin{array}{c}\text { Allocation } \\
\text { concealment }\end{array}$ & \begin{tabular}{|c|} 
Blinding of \\
participants \\
and personnel \\
\end{tabular} & $\begin{array}{l}\text { Blinding of } \\
\text { outcome } \\
\text { assessment }\end{array}$ & $\begin{array}{c}\text { Incomplete } \\
\text { outcome data } \\
\text { addresses } \\
\end{array}$ & $\begin{array}{l}\text { Selective } \\
\text { reporting }\end{array}$ & $\begin{array}{c}\text { Other } \\
\text { bias }\end{array}$ & $\begin{array}{c}\text { Overall } \\
\text { risk of } \\
\text { bias } \\
\end{array}$ \\
\hline Aimetti et al. [22] & Unclear & Unclear & Low & Low & Low & Low & Low & High \\
\hline Azizi and Moghaddam [23] & Low & Low & Unclear & High & Low & Low & High & High \\
\hline Barone et al. [24] & Low & Unclear & Unclear & Low & Low & Low & Low & Moderate \\
\hline Barone et al. [25] & Low & Low & Low & Low & Low & Unclear & Low & Moderate \\
\hline Borg et al. [26] & Low & Unclear & Unclear & Low & Low & Low & Low & Moderate \\
\hline Brownfield and Weltman [27] & Low & Low & Low & Unclear & Low & Low & Low & Moderate \\
\hline Cardaropoli et al. [28] & Unclear & Unclear & Unclear & Low & Low & Low & Low & High \\
\hline Cook and Mealey [29] & Low & Unclear & Unclear & Unclear & Low & High & Low & High \\
\hline Eskow and Mealey [30] & Low & Unclear & Unclear & Unclear & High & Low & Low & High \\
\hline Fotek et al. [31] & High & High & High & Low & High & Low & Low & High \\
\hline Hoang et al. [32] & Low & Unclear & Unclear & Unclear & Low & Low & High & High \\
\hline Iasella et al. [33] & Low & Unclear & High & Low & Low & Low & Low & High \\
\hline Iorio-Siciliano et al. [34] & Low & Low & Low & High & Low & Low & Low & Moderate \\
\hline Jung et al. [35] & Low & Low & Unclear & High & Low & Low & High & Moderate \\
\hline Jung et al. [36] & Low & Low & Low & Unclear & Low & Low & Low & Moderate \\
\hline Lim et al. [37] & Low & Low & Low & Low & Low & Low & Low & Low \\
\hline Mardas et al. [38] & Low & Low & Low & Low & Low & Low & Low & Low \\
\hline Meloni et al. [39] & Low & Unclear & Low & Low & Low & Low & Low & Low \\
\hline Nart et al. [40] & Low & Low & Low & Unclear & Low & Low & Low & Moderate \\
\hline Natto et al. [41] & Low & Low & Low & Low & Low & Low & Low & Low \\
\hline Pang et al. [42] & Unclear & Unclear & Unclear & High & Low & Low & Low & High \\
\hline Park et al. [43] & High & High & High & High & High & Low & Low & High \\
\hline Parashis et al. [44] & Low & Low & Unclear & Low & Low & Low & Low & Moderate \\
\hline Poulias et al. [45] & Low & Low & Unclear & Unclear & High & Low & Low & High \\
\hline Sadeghi et al. [46] & Low & Low & Unclear & Unclear & Low & Low & Low & High \\
\hline Toloue et al. [47] & Low & Low & Unclear & Unclear & Unclear & Low & Low & High \\
\hline Vance et al. [48] & Low & Unclear & Unclear & Low & High & High & Low & High \\
\hline Whetman et al. [49] & Low & Low & Unclear & Unclear & Low & Low & Low & Moderate \\
\hline Wood and Mealey [50] & Unclear & Unclear & Low & Low & Low & Low & Low & Moderate \\
\hline Serrano Mendez [51] & Low & Low & Low & Unclear & Low & Low & Low & Moderate \\
\hline Fernandes et al. [52] & Low & High & Low & Low & Low & Low & Low & Moderate \\
\hline Fernandes et al. [53] & Low & High & High & Low & Low & Low & Low & High \\
\hline Festa et al. [54] & Low & Unclear & Unclear & Unclear & Low & Low & Low & High \\
\hline Gholami et al. [55] & Low & Unclear & Low & Low & Low & Low & Low & Moderate \\
\hline Hassan et al. [56] & Low & Low & Low & Unclear & Low & Low & Low & Moderate \\
\hline Temmerman et al. [57] & Low & Low & Unclear & High & Low & Low & Low & High \\
\hline Kotsakis et al. [58] & Low & Unclear & Unclear & Low & Low & Low & High & High \\
\hline Guarnieri et al. [59] & Low & Low & Unclear & Low & Low & Low & Low & High \\
\hline Barone et al. [60] & Low & Low & Unclear & High & Low & Low & Low & High \\
\hline Demetter et al. [61] & Low & Low & Unclear & Unclear & Low & Low & Low & Moderate \\
\hline
\end{tabular}

with the use of xenogeneic, allogeneic, and alloplastic grafting materials, respectively. Additionally, the results in this review also confirm, although based on a limited sample of studies, that proximal sites of the socket exhibited less vertical dimensional reduction, compared to the mid-buccal and mid-lingual sites. And similarly, the horizontal resorption seems to be gradually minimized as the changes are evaluated further apical from the crest.

The magnitude and dynamics of the alveolar ridge's dimensional changes subsequent to tooth extraction are dictated and influenced by a variety of systemic and local factors, namely the extent of the traumatic injury during extraction, socket morphology, the presence of infection, smoking, the tooth type and position, the presence of periodontal disease, the hard and soft tissue phenotype, patient compliance, and most importantly, the number and thickness of the remaining intact socket walls. While this review failed to analyse the effect of such variables due to 
insufficient data and/or significant heterogeneity amongst the included studies, previous systematic reviews and meta-analyses have demonstrated a superior outcome in ridge preservation associated with baseline buccal bone thickness greater than $1 \mathrm{~mm}$ [8]. In contrast, a recent RCT concluded that ARP only influences the degree of ridge resorption at sites with $\leq 1 \mathrm{~mm}$ of buccal wall thickness [63].

The present investigation was able to analyse a large number of studies grouping the results based on the source of the bone substitutes used. While this method of managing the available data and the analysis of a large heterogenic sample present with inherent limitations, the results revealed similar trends across the included studies. As such, two main conclusions can be drawn: (1) as previously reported, ARP possesses the ability to diminish the resorption process following tooth extraction and (2) there are apparently only minimal differences between the bone substitutes. These results are in concordance with previous investigations reporting similar clinical outcomes associated with ARP using different bone substitutes $[\underline{30}, \underline{55}, \underline{61}]$. Despite this, there is a systematic review and meta-analysis that has reported superior outcomes ascribed to xenogeneic or allogeneic bone substitutes in combination with a collagen sponge or membrane []․ Despite minimal differences between the bone substitutes, the results of this review also favour both xenogeneic and allogeneic grafting with slightly less resultant resorption.

A primary limitation of the present investigation is the inclusion of multiple different grafting techniques and barrier membranes in the analysis. Another limitation includes several local and systemic factors known to play a role in the remodelling process that could not be evaluated. Also, the variation between the time points for evaluating the resorption process may have played a significant role in the reported outcomes. The weighted mean values of the different materials should be read and considered with caution as no statistical comparisons have been performed between the different treatment groups of bone grafts.

Finally, it is important to bear in mind that while ARP is most often performed in preparation for posterior implant placement, implant-related outcomes are often underreported in these investigations. As such, future studies should evaluate outcomes such as the feasibility of implant placement, the need for further grafting, as well as the long-term implant survival and success rates when placed into sockets previously grafted with different materials. Similarly, patientreported outcomes have rarely been investigated with regards to ARP.

\section{CONCLUSIONS}

Alveolar ridge preservation with the use of different bone substitutes represents an effective method for diminishing the physiological resorption process after tooth extraction. Additionally, minimal differences in resorption rate were observed between allogeneic, xenogeneic and alloplastic grafting materials.

\section{ACKNOWLEDGMENTS AND DISCLOSURE STATEMENTS}

The authors thank Dr. Shayan Barootchi (Department of Periodontics and Oral Medicine, University of Michigan School of Dentistry, Ann Arbor, Michigan, USA) for his assistance in conducting the statistical analysis.

\section{REFERENCES}

1. Buser D, Martin W, Belser UC. Optimizing esthetics for implant restorations in the anterior maxilla: anatomic and surgical considerations. Int J Oral Maxillofac Implants. 2004;19 Suppl:43-61. [Medline: 15635945]

2. Araújo MG, Silva CO, Misawa M, Sukekava F. Alveolar socket healing: what can we learn? Periodontol 2000. 2015 Jun;68(1):122-34. [Medline: 25867983] [doi: 10.1111/prd.12082]

3. Chappuis V, Araújo MG, Buser D. Clinical relevance of dimensional bone and soft tissue alterations post-extraction in esthetic sites. Periodontol 2000. 2017 Feb;73(1):73-83. [Medline: 28000281] [doi: 10.1111/prd.12167]

4. Schropp L, Wenzel A, Kostopoulos L, Karring T. Bone healing and soft tissue contour changes following singletooth extraction: a clinical and radiographic 12-month prospective study. Int J Periodontics Restorative Dent. 2003 Aug;23(4):313-23. [Medline: 12956475]

5. Van der Weijden F, Dell'Acqua F, Slot DE. Alveolar bone dimensional changes of post-extraction sockets in humans: a systematic review. J Clin Periodontol. 2009 Dec;36(12):1048-58. [Medline: 19929956] [doi: 10.1111/j.1600-051X.2009.01482.x]

6. Tan WL, Wong TL, Wong MC, Lang NP. A systematic review of post-extractional alveolar hard and soft tissue dimensional changes in humans. Clin Oral Implants Res. 2012 Feb;23 Suppl 5:1-21. [Medline: 22211303] [doi: 10.1111/j.1600-0501.2011.02375.x] 
7. Atieh MA, Alsabeeha NH, Payne AG, Duncan W, Faggion CM, Esposito M. Interventions for replacing missing teeth: alveolar ridge preservation techniques for dental implant site development. Cochrane Database Syst Rev. 2015 May 28;(5):CD010176. [Medline: 26020735] [PMC free article: 6464392] [doi: 10.1002/14651858.CD010176.pub2]

8. Avila-Ortiz G, Chambrone L, Vignoletti F. Effect of alveolar ridge preservation interventions following tooth extraction: A systematic review and meta-analysis. J Clin Periodontol. 2019 Jun;46 Suppl 21:195-223. [Medline: 30623987] [doi: 10.1111 jicpe.13057]

9. Mardas N, Trullenque-Eriksson A, MacBeth N, Petrie A, Donos N. Does ridge preservation following tooth extraction improve implant treatment outcomes: a systematic review: Group 4: Therapeutic concepts \& methods. Clin Oral Implants Res. 2015 Sep;26 Suppl 11:180-201. [Medline: 26078004] [doi: 10.1111/clr.12639]

10. MacBeth N, Trullenque-Eriksson A, Donos N, Mardas N. Hard and soft tissue changes following alveolar ridge preservation: a systematic review. Clin Oral Implants Res. 2017 Aug;28(8):982-1004. [Medline: 27458031] [doi: 10.1111/clr.12911]

11. Troiano G, Zhurakivska K, Lo Muzio L, Laino L, Cicciù M, Lo Russo L. Combination of bone graft and resorbable membrane for alveolar ridge preservation: A systematic review, meta-analysis, and trial sequential analysis. J Periodontol. 2018 Jan;89(1):46-57. [Medline: 28895779] [doi: 10.1902/jop.2017.170241]

12. Avila-Ortiz G, Elangovan S, Kramer KW, Blanchette D, Dawson DV. Effect of alveolar ridge preservation after tooth extraction: a systematic review and meta-analysis. J Dent Res. 2014 Oct;93(10):950-8 [Medline: 24966231] [PMC free article: 4293706 ] [doi: 10.1177/0022034514541127]

13. Bassir SH, Alhareky M, Wangsrimongkol B, Jia Y, Karimbux N. Systematic Review and Meta-Analysis of Hard Tissue Outcomes of Alveolar Ridge Preservation. Int J Oral Maxillofac Implants. 2018 Sep/Oct;33(5):979-994. [Medline: 30231083] [doi: 10.11607/jomi.6399]

14. Iocca O, Farcomeni A, Pardiñas Lopez S, Talib HS. Alveolar ridge preservation after tooth extraction: a Bayesian Network meta-analysis of grafting materials efficacy on prevention of bone height and width reduction. J Clin Periodontol. 2017 Jan;44(1):104-114. [Medline: 27712001] [doi: 10.1111/jcpe.12633]

15. Willenbacher M, Al-Nawas B, Berres M, Kämmerer PW, Schiegnitz E. The Effects of Alveolar Ridge Preservation: A Meta-Analysis. Clin Implant Dent Relat Res. 2016 Dec;18(6):1248-1268. [Medline:26132885] [doi: 10.1111/cid.12364]

16. Vignoletti F, Matesanz P, Rodrigo D, Figuero E, Martin C, Sanz M. Surgical protocols for ridge preservation after tooth extraction. A systematic review. Clin Oral Implants Res. 2012 Feb;23 Suppl 5:22-38. [Medline: 22211304] [doi: 10.1111/j.1600-0501.2011.02331.x]

17. De Risi V, Clementini M, Vittorini G, Mannocci A, De Sanctis M. Alveolar ridge preservation techniques: a systematic review and meta-analysis of histological and histomorphometrical data. Clin Oral Implants Res. 2015 Jan;26(1):50-68. [Medline: 27007188] [doi: 10.1111/clr.12288]

18. Thoma DS, Naenni N, Figuero E, Hämmerle CHF, Schwarz F, Jung RE, Sanz-Sánchez I. Effects of soft tissue augmentation procedures on peri-implant health or disease: A systematic review and meta-analysis. Clin Oral Implants Res. 2018 Mar;29 Suppl 15:32-49. [Medline: 29498129] [doi: 10.1111/clr.13114]

19. Moher D, Liberati A, Tetzlaff J, Altman DG; PRISMA Group. Preferred reporting items for systematic reviews and meta-analyses: the PRISMA statement. J Clin Epidemiol. 2009 Oct;62(10):1006-12. [Medline: 19631508] [doi: 10.1016/j.jclinepi.2009.06.005]

20. Shea BJ, Hamel C, Wells GA, Bouter LM, Kristjansson E, Grimshaw J, Henry DA, Boers M. AMSTAR is a reliable and valid measurement tool to assess the methodological quality of systematic reviews. J Clin Epidemiol. 2009 Oct;62(10):1013-20. [Medline: 19230606] [doi: 10.1016/j.jclinepi.2008.10.009]

21. Higgins JP, Altman DG, Gøtzsche PC, Jüni P, Moher D, Oxman AD, Savovic J, Schulz KF, Weeks L, Sterne JA; Cochrane Bias Methods Group; Cochrane Statistical Methods Group. The Cochrane Collaboration's tool for assessing risk of bias in randomised trials. BMJ. 2011 Oct 18;343:d5928. [Medline: 22008217] [PMC free article: 3196245] [doi: 10.1136/bmj.d5928]

22. Aimetti M, Romano F, Griga FB, Godio L. Clinical and histologic healing of human extraction sockets filled with calcium sulfate. Int J Oral Maxillofac Implants. 2009 Sep-Oct;24(5):902-9. [Medline: 19865631]

23. Azizi A, Moghaddam SA, A study on the effect of Bio-Oss and collagen membrane on the repair of dental socket. J Isfahan Dent Sch. 2009;5(3):133-9.

24. Barone A, Aldini NN, Fini M, Giardino R, Calvo Guirado JL, Covani U. Xenograft versus extraction alone for ridge preservation after tooth removal: a clinical and histomorphometric study. J Periodontol. 2008 Aug;79(8):1370-7. [Medline: 18672985] [doi: 10.1902/jop.2008.070628]

25. Barone A, Toti P, Piattelli A, Iezzi G, Derchi G, Covani U. Extraction socket healing in humans after ridge preservation techniques: comparison between flapless and flapped procedures in a randomized clinical trial. J Periodontol. 2014 Jan;85(1):14-23. [Medline: 23688103] [doi: 10.1902/jop.2013.120711]

26. Borg TD, Mealey BL. Histologic healing following tooth extraction with ridge preservation using mineralized versus combined mineralized-demineralized freeze-dried bone allograft: a randomized controlled clinical trial. J Periodontol. 2015 Mar;86(3):348-55. [Medline: 25415247] [doi: 10.1902/jop.2014.140483] 
27. Brownfield LA, Weltman RL. Ridge preservation with or without an osteoinductive allograft: a clinical, radiographic, micro-computed tomography, and histologic study evaluating dimensional changes and new bone formation of the alveolar ridge. J Periodontol. 2012 May;83(5):581-9. [Medline: 21942791] [doi: 10.1902/jop.2011.110365]

28. Cardaropoli D, Tamagnone L, Roffredo A, Gaveglio L. Relationship between the buccal bone plate thickness and the healing of postextraction sockets with/without ridge preservation. Int J Periodontics Restorative Dent. 2014 Mar-Apr;34(2):211-7. [Medline: 24600657] [doi: 10.11607/prd.1885]

29. Cook DC, Mealey BL. Histologic comparison of healing following tooth extraction with ridge preservation using two different xenograft protocols. J Periodontol. 2013 May;84(5):585-94. [Medline: 22680300] [doi: 10.1902/jop.2012.120219]

30. Eskow AJ, Mealey BL. Evaluation of healing following tooth extraction with ridge preservation using cortical versus cancellous freeze-dried bone allograft. J Periodontol. 2014 Apr;85(4):514-24. [Medline: 23725026] [doi: 10.1902/jop.2013.130178]

31. Fotek PD, Neiva RF, Wang HL. Comparison of dermal matrix and polytetrafluoroethylene membrane for socket bone augmentation: a clinical and histologic study. J Periodontol. 2009 May;80(5):776-85. [Medline: 19405831] [doi: 10.1902/jop.2009.080514]

32. Hoang TN, Mealey BL. Histologic comparison of healing after ridge preservation using human demineralized bone matrix putty with one versus two different-sized bone particles. J Periodontol. 2012 Feb;83(2):174-81. [Medline: 21692633] [doi: 10.1902/jop.2011.110209]

33. Iasella JM, Greenwell H, Miller RL, Hill M, Drisko C, Bohra AA, Scheetz JP. Ridge preservation with freeze-dried bone allograft and a collagen membrane compared to extraction alone for implant site development: a clinical and histologic study in humans. J Periodontol. 2003 Jul;74(7):990-9. [Medline: 12931761] [doi: 10.1902/jop.2003.74.7.990]

34. Iorio-Siciliano V, Blasi A, Nicolò M, Iorio-Siciliano A, Riccitiello F, Ramaglia L. Clinical Outcomes of Socket Preservation Using Bovine-Derived Xenograft Collagen and Collagen Membrane Post-Tooth Extraction: A 6-Month Randomized Controlled Clinical Trial. Int J Periodontics Restorative Dent. 2017 Sep/Oct;37(5):e290-e296. [Medline: 28817143] [doi: 10.11607/prd.2474]

35. Jung RE, Philipp A, Annen BM, Signorelli L, Thoma DS, Hämmerle CH, Attin T, Schmidlin P. Radiographic evaluation of different techniques for ridge preservation after tooth extraction: a randomized controlled clinical trial. J Clin Periodontol. 2013 Jan;40(1):90-8. [Medline: 23163915] [doi: 10.1111/jcpe.12027]

36. Jung RE, Sapata VM, Hämmerle CHF, Wu H, Hu XL, Lin Y. Combined use of xenogeneic bone substitute material covered with a native bilayer collagen membrane for alveolar ridge preservation: A randomized controlled clinical trial. Clin Oral Implants Res. 2018 May;29(5):522-529. [Medline: 29607553] [doi: 10.1111/clr.13149]

37. Lim HC, Jung UW, You H, Lee JS. Randomized clinical trial of ridge preservation using porcine bone/cross-linked collagen vs. bovine bone/non-cross-linked collagen: cone beam computed tomographic analysis. Clin Oral Implants Res. 2017 Dec;28(12):1492-1500. [Medline: 28370361] [doi: 10.1111/clr.13017]

38. Mardas N, Chadha V, Donos N. Alveolar ridge preservation with guided bone regeneration and a synthetic bone substitute or a bovine-derived xenograft: a randomized, controlled clinical trial. Clin Oral Implants Res. 2010 Jul;21(7):688-98. [Medline: 20636724] [doi: 10.1111/j.1600-0501.2010.01918.x]

39. Meloni SM, Tallarico M, Lolli FM, Deledda A, Pisano M, Jovanovic SA. Postextraction socket preservation using epithelial connective tissue graft vs porcine collagen matrix. 1-year results of a randomised controlled trial. Eur J Oral Implantol. 2015 Spring;8(1):39-48. [Medline: 25738178]

40. Nart J, Barallat L, Jimenez D, Mestres J, Gómez A, Carrasco MA, Violant D, Ruíz-Magaz V. Radiographic and histological evaluation of deproteinized bovine bone mineral vs. deproteinized bovine bone mineral with $10 \%$ collagen in ridge preservation. A randomized controlled clinical trial. Clin Oral Implants Res. 2017 Jul;28(7):840-848. [Medline: 27335267] [doi: 10.1111/clr.12889]

41. Natto ZS, Parashis A, Steffensen B, Ganguly R, Finkelman MD, Jeong YN. Efficacy of collagen matrix seal and collagen sponge on ridge preservation in combination with bone allograft: A randomized controlled clinical trial. J Clin Periodontol. 2017 Jun;44(6):649-659. [Medline: 28303642] [doi: 10.1111/jepe.12722]

42. Pang C, Ding Y, Zhou H, Qin R, Hou R, Zhang G, Hu K. Alveolar ridge preservation with deproteinized bovine bone graft and collagen membrane and delayed implants. J Craniofac Surg. 2014 Sep;25(5):1698-702. [Medline: 25148644] [doi: 10.1097/SCS.0000000000000887]

43. Park JC, Koo KT, Lim HC. The hidden X suture: a technical note on a novel suture technique for alveolar ridge preservation. J Periodontal Implant Sci. 2016 Dec;46(6):415-425. [Medline: 28050319] [PMC free article: 5200867] [doi: 10.5051/jpis.2016.46.6.415]

44. Parashis AO, Hawley CE, Stark PC, Ganguly R, Hanley JB, Steffensen B. Prospective Clinical and Radiographic Study of Alveolar Ridge Preservation Combining Freeze-Dried Bone Allograft With Two Xenogeneic Collagen Matrices. J Periodontol. 2016 Apr;87(4):416-25. [Medline: 26745614] [doi: 10.1902/jop.2016.150500]

45. Poulias E, Greenwell H, Hill M, Morton D, Vidal R, Shumway B, Peterson TL. Ridge preservation comparing socket allograft alone to socket allograft plus facial overlay xenograft: a clinical and histologic study in humans. J Periodontol. 2013 Nov;84(11):1567-75. [Medline: 23339378] [doi: 10.1902/jop.2013.120585] 
46. Sadeghi R, Babaei M, Miremadi SA, Abbas FM. A randomized controlled evaluation of alveolar ridge preservation following tooth extraction using deproteinized bovine bone mineral and demineralized freeze-dried bone allograft. Dent Res J (Isfahan). 2016 Mar-Apr;13(2):151-9. [Medline: 27076830] [PMC free article: 4810913] [doi: $10.4103 / 1735-3327.178202]$

47. Toloue SM, Chesnoiu-Matei I, Blanchard SB. A clinical and histomorphometric study of calcium sulfate compared with freeze-dried bone allograft for alveolar ridge preservation. J Periodontol. 2012 Jul;83(7):847-55. [Medline: 22166162] [doi: 10.1902/jop.2011.110470]

48. Vance GS, Greenwell H, Miller RL, Hill M, Johnston H, Scheetz JP. Comparison of an allograft in an experimental putty carrier and a bovine-derived xenograft used in ridge preservation: a clinical and histologic study in humans. Int J Oral Maxillofac Implants. 2004 Jul-Aug;19(4):491-7. [Medline: 15346745]

49. Whetman J, Mealey BL. Effect of Healing Time on New Bone Formation After Tooth Extraction and Ridge Preservation With Demineralized Freeze-Dried Bone Allograft: A Randomized Controlled Clinical Trial. J Periodontol. 2016 Sep;87(9):1022-9. [Medline: 27133791] [doi: 10.1902/jop.2016.160139]

50. Wood RA, Mealey BL. Histologic comparison of healing after tooth extraction with ridge preservation using mineralized versus demineralized freeze-dried bone allograft. J Periodontol. 2012 Mar;83(3):329-36. [Medline: 21749166] [doi: 10.1902/jop.2011.110270]

51. Serrano Méndez CA, Lang NP, Caneva M, Ramírez Lemus G, Mora Solano G, Botticelli D. Comparison of allografts and xenografts used for alveolar ridge preservation. A clinical and histomorphometric RCT in humans. Clin Implant Dent Relat Res. 2017 Aug;19(4):608-615. [Medline: 28466494] [doi: 10.1111/cid.12490]

52. Fernandes PG, Muglia VA, Reino DM, Maia LP, de Moraes Grisi MF, de Souza SL, Taba M Jr, Palioto DB, de Almeida AG, Novaes AB Jr. Socket Preservation Therapy with Acellular Dermal Matrix and Mineralized Bone Allograft After Tooth Extraction in Humans: A Clinical and Histomorphometric Study. Int J Periodontics Restorative Dent. 2016 Mar-Apr;36(2):e16-25. [Medline: 26901306] [doi: 10.11607/prd.2307]

53. Fernandes PG, Novaes AB Jr, de Queiroz AC, de Souza SL, Taba M Jr, Palioto DB, Grisi MF. Ridge preservation with acellular dermal matrix and anorganic bone matrix cell-binding peptide P-15 after tooth extraction in humans. J Periodontol. 2011 Jan;82(1):72-9. [Medline: 20722531] [doi: 10.1902/jop.2010.100241]

54. Festa VM, Addabbo F, Laino L, Femiano F, Rullo R. Porcine-derived xenograft combined with a soft cortical membrane versus extraction alone for implant site development: a clinical study in humans. Clin Implant Dent Relat Res. 2013 Oct;15(5):707-13. [Medline: 22082037] [doi: 10.1111/j.1708-8208.2011.00398.x]

55. Gholami, G.A., et al., Clinical, histologic and histomorphometric evaluation of socket preservation using a synthetic nanocrystalline hydroxyapatite in comparison with a bovine xenograft: a randomized clinical trial. Clin Oral Implants Res, 2012. 23(10): p. 1198-204. [Medline: 22092485] [doi: 10.1111/j.1600-0501.2011.02288.x]

56. Hassan M, Prakasam S, Bain C, Ghoneima A, Liu SS. A Randomized Split-Mouth Clinical Trial on Effectiveness of Amnion-Chorion Membranes in Alveolar Ridge Preservation: A Clinical, Radiologic, and Morphometric Study. Int J Oral Maxillofac Implants. 2017 Nov/Dec;32(6):1389-1398. [Medline: 29140383] [doi: 10.11607/jomi.5875]

57. Temmerman A, Vandessel J, Castro A, Jacobs R, Teughels W, Pinto N, Quirynen M. The use of leucocyte and platelet-rich fibrin in socket management and ridge preservation: a split-mouth, randomized, controlled clinical trial. J Clin Periodontol. 2016 Nov;43(11):990-999. [Medline: 27509214] [doi: 10.1111/jepe.12612]

58. Kotsakis GA, Salama M, Chrepa V, Hinrichs JE, Gaillard P. A randomized, blinded, controlled clinical study of particulate anorganic bovine bone mineral and calcium phosphosilicate putty bone substitutes for socket preservation. Int J Oral Maxillofac Implants. 2014 Jan-Feb;29(1):141-51. [Medline: 24451865] [doi: 10.11607/jomi.3230]

59. Guarnieri R, Testarelli L, Stefanelli L, De Angelis F, Mencio F, Pompa G, Di Carlo S. Bone Healing in Extraction Sockets Covered With Collagen Membrane Alone or Associated With Porcine-Derived Bone Graft: a Comparative Histological and Histomorphometric Analysis. J Oral Maxillofac Res. 2017 Dec 31;8(4):e4. [Medline: 29435206] [PMC free article: 5806041] [doi: 10.5037/jomr.2017.8404]

60. Barone A, Toti P, Quaranta A, Alfonsi F, Cucchi A, Negri B, Di Felice R, Marchionni S, Calvo-Guirado JL, Covani U, Nannmark U. Clinical and Histological changes after ridge preservation with two xenografts: preliminary results from a multicentre randomized controlled clinical trial. J Clin Periodontol. 2017 Feb;44(2):204-214. [Medline: 27883211] [doi: $10.1111 /$ jcpe.12655]

61. Demetter RS, Calahan BG, Mealey BL. Histologic Evaluation of Wound Healing After Ridge Preservation With Cortical, Cancellous, and Combined Cortico-Cancellous Freeze-Dried Bone Allograft: A Randomized Controlled Clinical Trial. J Periodontol. 2017 Sep;88(9):860-868. [Medline: 28452622] [doi: 10.1902/jop.2017.170155]

62. Jambhekar S, Kernen F, Bidra AS. Clinical and histologic outcomes of socket grafting after flapless tooth extraction: a systematic review of randomized controlled clinical trials. J Prosthet Dent. 2015 May;113(5):371-82. [Medline: 25749077] [doi: 10.1016/j.prosdent.2014.12.009]

63. Tomasi C, Donati M, Cecchinato D, Szathvary I, Corrà E, Lindhe J. Effect of socket grafting with deproteinized bone mineral: An RCT on dimensional alterations after 6 months. Clin Oral Implants Res. 2018 May;29(5):435-442. [Medline: 29532525] [doi: 10.1111/clr.13141] 


\section{To cite this article:}

Majzoub J, Ravida A, Starch-Jensen T, Tattan M, Suárez-López del Amo F.

The Influence of Different Grafting Materials on Alveolar Ridge Preservation: a Systematic Review

J Oral Maxillofac Res 2019;10(3):e6

URL: http://www.ejomr.org/JOMR/archives/2019/3/e6/v10n3e6.pdf

doi: $\underline{10.5037 / j o m r .2019 .10306}$

Copyright (C) Majzoub J, Ravida A, Starch-Jensen T, Tattan M, Suárez-López del Amo F. Published in the JOURNAL OF ORAL \& MAXILLOFACIAL RESEARCH (http://www.ejomr.org), 5 September 2019.

This is an open-access article, first published in the JOURNAL OF ORAL \& MAXILLOFACIAL RESEARCH, distributed under the terms of the Creative Commons Attribution-Noncommercial-No Derivative Works 3.0 Unported License, which permits unrestricted non-commercial use, distribution, and reproduction in any medium, provided the original work and is properly cited. The copyright, license information and link to the original publication on (http://www.ejomr.org) must be included. 